\title{
CINCUENTA AÑOS DESPUÉS DE LA SEGUNDA EDICIÓN DE LA REINE RECHTSLEHRE. SOBRE EL TRASFONDO DE LA TEORÍA PURA DEL DERECHO Y SOBRE LO QUE QUEDA DE ELLA *
}

\author{
Juan Ruiz Manero \\ Universidad de Alicante
}

RESUMEN. Este texto se ocupa de dos cuestiones relacionadas con la teoría kelseniana del Derecho: en primer lugar, del trasfondo que explica los rasgos centrales de esa misma teoría; en segundo lugar, de lo que sigue vivo y lo que debe considerarse muerto en la construcción de KELSEN.

A juicio del autor, los elementos más relevantes del trasfondo de la teoría kelseniana son una justificación grosso modo hobbesiana del Derecho, por un lado, y la tesis de la imposibilidad de la razón práctica, por otro.

También a juicio del autor, la mayor parte de las articulaciones concretas de la teoría kelseniana deben considerarse hoy como muertas. Lo que no impide conceder la máxima importancia histórica a la aportación de KELSEN y entender que su ambición de articular el discurso sobre el Derecho sobre la base de una teoría que merezca tal nombre debe considerarse como completamente actual.

Palabras clave: KELSEN, teoría pura del Derecho.

ABSTRACT. This text deals with two issues related to KELSEN's theory of law: firstly, with the background which explains the core features of that theory and secondly, with what is still alive and what should be considered dead in KELSEN's construction.

According to the author, the most important elements in the background of KELSEN's theory are a roughly Hobbesian justification of law and the thesis of the impossibility of practical reason.

The author also holds that most of the specific kelsenian theses should now be regarded as dead. This does not preclude the granting of a great historical importance to KELSEN'S contribution and the understanding that his ambition to articulate discourse on the law on the basis of a theory worthy of the name should be regarded as completely valid today.

Keywords: KELSEN, pure theory of Law.

* Fecha de recepción: 20 de enero de 2011. Fecha de aceptación: 20 de febrero de 2011.

Versión revisada de la intervención, en respuesta a U. SCHMILL, en el acto de clausura de la 7. a edición del curso de especialista en argumentación jurídica (Alicante, junio 2010). 


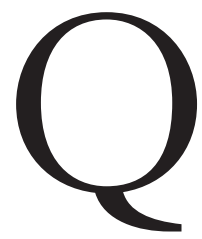

uisiera empezar mi intervención expresando mi satisfacción por compartir esta mesa con U. Schmill. U. Schmill es uno de los filósofos del Derecho más eminentes de nuestra lengua, es uno de los mejores conocedores de KELSEN, como ha mostrado la exposición que acaba de hacer, y es, sin ninguna duda, el más ilustre de nuestros kelsenianos actuales. SCHMill ha presentado el conjunto de la imponente producción kelseniana. Yo, por mi parte, me voy a limitar a su teoría del Derecho. Teoría del Derecho que no voy a presentar, sino que voy a dar por conocida y, en relación con la cual voy a ocuparme de dos aspectos: en un primer apartado, voy a abordar la cuestión del trasfondo que, en mi opinión, explica los rasgos centrales de la teoría kelseniana; en un segundo apartado, voy a tratar de determinar qué es lo que hoy puede considerarse vivo o muerto de esa misma teoría.

\section{1.}

Por lo que hace a la primera cuestión puede ser útil partir de una conocidísima dicotomía bobbiana, la que distingue entre filosofías del Derecho de los filósofos y filosofías del Derecho de los juristas ${ }^{1}$. La filosofía del Derecho de KELSEN es, obvio es decirlo, una filosofía del Derecho de jurista. Y ello, también es obvio decirlo, no sólo ni tanto en el sentido de que KELSEN fuera profesionalmente un jurista, sino en el sentido de que su filosofía del Derecho parte de, y trata de responder a, problemas propios de jurista. Y, más precisamente, a problemas propios de jurista teórico, de profesor de Derecho. La filosofía jurídica kelseniana constituye, señaló M. LOSANO, un opus perpetuum en el sentido de que todos los trabajos en los que KELSEN fue desarrollando la teoría pura del Derecho a lo largo de sesenta años no sólo muestran a esta teoría en un estado de «continua transformación», sino que también constituyen una «obra intrínsecamente unitaria a través del tiempo» ${ }^{2}$. Podríamos decir que, desde los Problemas capitales de la teoría del Derecho público de 1911 hasta la póstuma Teoría general de las normas, pasando por las diversas ediciones de la Teoría pura del Derecho o por la Teoría general del Derecho y del Estado, KELSEN no dejó de reescribir, una y otra vez, en cierto sentido, el mismo libro. Enriqueciéndolo, desde luego, mediante desarrollos, integraciones de influencias diferentes y rectificaciones. Estos desarrollos, integraciones y rectificaciones permiten trazar periodizaciones de la obra kelseniana tan bien construidas y tan bien fundadas como la que ha elaborado, por ejemplo, P. CHIASSO$\mathrm{NI}^{3}$. Pero estos sucesivos desarrollos, integraciones y rectificaciones, van dando lugar a lo que podemos considerar, en el fondo, como versiones diferentes del mismo libro. Pues bien: ya en los Problemas capitales de 1911 encontramos lo que constituye, a mi juicio, el quicio más importante para entender las pretensiones y la orientación central de la teoría pura del Derecho a lo largo de todo su itinerario. Que viene a ser, a mi

\footnotetext{
${ }^{1}$ N. Boвbio, «Naturaleza y función de la filosofía del Derecho» (1962), en BobBIO, Contribución a la teoría del Derecho, ed. de A. Ruiz Miguel, Fernando Torres Editor, Valencia, 1980, 91 y ss.

2 M. Losano, «La teoría pura del Derecho del logicismo al irracionalismo», trad. de J. Ruiz Manero, en Doxa, núm. 2, 1985, 57.

3 P. Chiassoni, L'utopia della ragione analitica. Origini, oggetti e metodi della filosofia del diritto positivo, Giappichelli, Torino, 2005, 58 y ss.; también: L'indirizzo analitico nella filosofia del diritto. I Da Bentham a Kelsen, Giappichelli, Torino, 2009, 306 y ss.
} 
juicio, la siguiente: KELSEN pretende fundamentar y dar mayor rigor a algo que sustancialmente acepta, esto es, los rasgos centrales de la visión del trabajo del jurista teórico tal como aparecen en la doctrina alemana del Derecho público y, emblemáticamente, en la obra de JELLINEK. Y es que, en efecto, ya en la obra de LABAND y, sobre todo, en la de JeLLINEK, el trabajo del jurista aparece como un trabajo de conocimiento de normas en cuanto normas, y no de hechos, y un conocimiento que se presenta como neutral desde el punto de vista de las valoraciones ético-políticas. Lo que hará KELSEN es fundamentar filosóficamente y, con ello, estilizar y dar mayor rigor y radicalidad a la concepción de una ciencia del Derecho construida sobre estos moldes. Y el neokantismo es la herramienta, adecuada para esta tarea, que encuentra en el mercado filosófico del momento; en fases posteriores, el neokantismo coexistirá con el positivismo lógico; pero neokantismo y positivismo lógico son para KELSEN, a mi juicio, meras herramientas externas que toma y utiliza en la medida en que son útiles para los problemas que, como jurista teórico, le preocupan.

¿Y por qué KELSEN opta por un modelo de ciencia jurídica como el que se prescribe, con sólo muy leves cambios de acento a lo largo de más de sesenta años, en las sucesivas versiones de la teoría pura? Quiero decir, por un modelo de ciencia jurídica que trata de «obtener solamente un conocimiento orientado hacia el Derecho», del que debe quedar excluido todo aquello que «no pertenece al objeto precisamente determinado como jurídico» ${ }^{4}$, vale decir, del que deben quedar excluidos tanto los enunciados basados en el principio de causalidad — los enunciados relativos a relaciones entre hechos- como los enunciados que expresan valoraciones externas al Derecho mismo: el discurso de la ciencia del Derecho debe estar integrado exclusivamente por enunciados que constituyan «una descripción axiológicamente adiáfora de su objeto [...] sin referencia a ningún valor metajurídico, y sin ninguna aprobación ni desaprobación emotiva» ${ }^{5}$. Si entendemos que en esta doble exclusión — de juicios causales y de juicios de valor - es donde radica centralmente la pureza del modelo de ciencia jurídica prescrito por KELSEN, dar las razones de esta doble exclusión equivaldría a explicar el trasfondo que determina la Reinheit, por así decirlo, de la Rechtslebre kelseniana. Pues bien, a mi modo de ver, este trasfondo está constituido básicamente por dos elementos: en primer lugar, por una justificación del Derecho que, desde luego grosso modo y desde luego también con todas las cautelas que se consideren debidas, podemos calificar como hobbesiana; en segundo lugar, por la tesis de la imposibilidad de la razón práctica, es decir, de la no posibilidad de fundamentación racional de los juicios de valor. La suma de estos dos elementos determina la justificación de un discurso sobre el Derecho que vea este exclusivamente como orden normativo, que trate de describirlo como tal y que excluya de su horizonte cualquier valoración externa de ese mismo orden normativo.

Y es que toda la polémica de KELSEN sobre el iusnaturalismo, toda su insistencia en la imposibilidad de fundamentar enunciados de deber sobre la base de enunciados de ser, no debe ocultar el hecho de que, de forma difícilmente compatible con ello, se encuentra asimismo en KELSEN una fundamentación del carácter justificado del Derecho, del carácter justificado de un orden coercitivo, que apela, en la mejor

${ }^{4}$ H. KelSEN, Teoría pura del Derecho (2. ${ }^{a}$ ed., 1960), trad. de R. J. Vernengo, México, UNAM, 1982, 15.

5 Ibid., 94. 
tradición hobbesiana, a características permanentes de la naturaleza humana. Así, por ejemplo, en sus polémicas con los marxistas, insiste una y otra vez en que «lo que verdaderamente hace necesario un ordenamiento coercitivo» no es, como los marxistas sostienen, la explotación económica de clase, sino ciertas constantes de la naturaleza humana que, siendo mucho más básicas, permanecerían como tales aun en ausencia de esa explotación: se trata de «la oposición existente entre el ordenamiento social y los instintos, deseos e intereses de los hombres cuyo comportamiento es regulado por el ordenamiento» ${ }^{6}$. Confiar a este respecto, como hacen los marxistas, «en la completa transformación del hombre», una vez suprimida la explotación económica, viene a ser, a juicio de KELSEN, «el ejemplo escolástico de una utopía "no científica", por no estar basada en experiencia alguna» ${ }^{7}$. La realidad es que «el sexo y la ambición representan un papel [en la perturbación del orden social] por lo menos tan importante como las circunstancias económicas» ${ }^{8}$ y que no «existen divergencias de opinión [en campos como el religioso, el artístico o el erótico] que no puedan convertirse en un contraste de vida o muerte» ${ }^{9}$. Siendo así la naturaleza humana, el Derecho como orden coactivo es el único instrumento que puede instituir un orden «objetivo», situado por encima de la voluntad de los sujetos implicados en cada momento; el Derecho es la única alternativa al imperio de la anarquía o de la dominación por medio de la fuerza desnuda. Y el Derecho puede constituir operativamente tal alternativa sólo si su carácter normativo no se encuentra condicionado por la concordancia de sus contenidos con ningún patrón de justicia externo al mismo. Pues, si no es posible — como no lo es, a juicio de KELSEN- determinar objetivamente qué sea lo justo, si la justicia es un ideal irracional y los juicios sobre ella son pura expresión de preferencias no justificables, entonces subordinar la obligatoriedad del Derecho a su justicia supone que el Derecho no pueda cumplir su función de instauración de orden. Pues subordinar la obligatoriedad del Derecho a su justicia implicaría subordinar dicha obligatoriedad a los juicios subjetivos no concordantes y no justificables de cada uno y, así, abocar a la sociedad a una situación de lucha no normada entre los diversos individuos y grupos que sostienen juicios divergentes sobre la justicia.

En definitiva, a mi juicio, lo que late en el fondo de la construcción de KELSEN es que un discurso que describa el Derecho como normatividad, y no como algo situado en el plano de los hechos, y que no condicione esa normatividad a la concordancia de sus contenidos con algún patrón de justicia, es un discurso que coadyuva a la operatividad del Derecho como técnica de organización social, como técnica de instauración de orden. Y éste es, a mi juicio, el trasfondo relevante de la «pureza» prescrita por KELSEN para la ciencia jurídica. En este sentido, podríamos decir, usando terminología correspondiente a discusiones actuales, que KELSEN no es sólo un positivista excluyente, sino también, y esto ocupa un lugar más básico en su pensamiento, un positivista ético.

${ }^{6}$ H. KELSEN, La teoria generale del diritto e il materialismo storico (1931), trad. italiana de F. Riccobono, Roma, Istituto della Enciclopedia Italiana, 1979, 83.

7 H. Kelsen, Socialismo y Estado. Una investigación sobre la teoría política del marxismo (2." ed., 1923), trad. de A. García Ruiz, México, Siglo XXI, 1982, 220.

8 H. Kelsen: La teoría comunista del Derecho (1955), en KelsEn: Teoría comunista del Derecho y del Estado, trad. de A. J. Weiss, Buenos Aires, Emecé Editores, 1957, 65.

9 H. Kelsen, Socialismo y Estado, cit., 273. 
No ignoro que presentar así las cosas choca con la imagen habitual de la empresa kelseniana, en la que esta empresa aparece dominada por intereses puramente cognoscitivos, y no prácticos. Y puede pensarse que mi interpretación choca también con la constante insistencia de KELSEN en que el Derecho puede tener cualquier contenido porque puede perseguir cualquier finalidad. Esta última dificultad, sin embargo, es, a mi juicio, puramente aparente. Hace ya muchos años que BOBBIO, al que vuelvo otra vez, señaló que una reconstrucción adecuada de KELSEN tiene que integrar la consideración de que «si se admite que el Derecho puede servir para alcanzar los fines más diversos, pero al mismo tiempo se admite que el fin del orden no puede alcanzarse más que por medio del Derecho, el Derecho no es solamente un medio, sino que tiene un fin, o mejor dicho, es un medio para un fin específico» ${ }^{10}$.

La imagen habitual de acuerdo con la cual la empresa kelseniana obedecería exclusivamente a intereses cognoscitivos y no prácticos tiene, desde luego, importantes puntos de apoyo textuales en el propio KELSEN. Pero, frente a ello, puedo señalar que, a mi juicio, ubicar en primer plano los intereses prácticos permite presentar el conjunto de la construcción kelseniana con una mayor coherencia de sentido. Y puedo señalar también que, aunque esta interpretación mía no es la habitual, tampoco es original. Hace más de cincuenta años que U. SCARPELLI apuntaba en un sentido similar al escribir que cuando KELSEN «habla de la legalidad como el único sentido del valor justicia en que este valor interviene en los ordenamientos jurídicos positivos, parece situarse en un plano deontológico, presentando la teoría del Derecho como la preceptística a adoptar para asegurar el valor específico del Derecho, es decir, el orden» ${ }^{11}$ (310). Esto está escrito seis años antes de la publicación de la segunda edición de la Reine Rechtslebre. Hoy, cuando conmemoramos el cincuenta aniversario de la publicación de esta obra, no creo que podamos decirlo mejor.

\section{2.}

En mi opinión, si queremos deslindar adecuadamente lo que aún hoy está vivo y lo que está muerto del legado kelseniano, hay que empezar por reconocer que las articulaciones concretas de la teoría pura del Derecho pueden considerarse hoy, casi todas ellas, como muertas. Pero ello no implica minusvaloración alguna del papel histórico de la teoría kelseniana ni impide considerar que hay una lección de la misma que conserva hoy día toda su virtualidad y toda su frescura. De esto, de lo que está vivo en la teoría de KELSEN, me ocuparé al final de mi intervención.

\subsection{Lo que está muerto}

Empezaré por aquello que, a mi juicio, está muerto. Y para ello mostraré, mediante cuatro acotaciones críticas que no pretenden originalidad ninguna, lo que considero otros tantos puntos de fracaso capitales de la teoría kelseniana. La primera de estas acotaciones

${ }^{10}$ N. BobBIO, «Hacia una teoría funcional del Derecho», en AAVV: Derecho, filosofía y lenguaje. Homenaje a Ambrosio L. Gioja, Buenos Aires, Astrea, 1976, 13.

${ }_{11}$ U. SCARPELLI, «Società e natura nel pensiero di Hans Kelsen» (1954), en SCARPELLI, L'etica senza verità, Bologna, Il Mulino, 1982, 310. 
afecta, a propósito de la distinción entre multas e impuestos, a un aspecto de la pureza kelseniana y al lugar que en la misma ocupa el concepto de sanción; la segunda, a propósito de la identificación de las antinomias, a la tesis de que las normas genuinas o primarias son normas dirigidas exclusivamente a los órganos; la tercera acotación afecta al problema del conflicto entre normas de diferente jerarquía y, en relación con él, a la doctrina de la cláusula alternativa tácita; la cuarta, finalmente, versa sobre la doctrina de los enunciados descriptivos de deber ser. Las dos primeras de estas acotaciones muestran, en mi opinión, otros tantos fracasos de la teoría pura para proporcionarnos una imagen adecuada del Derecho; las acotaciones tercera y cuarta, por su parte, muestran cómo la construcción de KELSEN resulta fallida en relación con sus propias orientaciones programáticas.

Veamos todo ello.

\subsubsection{Primera acotación}

Esta primera acotación afecta a la prescripción kelseniana de no mirar jamás al mundo de los hechos para elucidar el sentido de las normas. Esta prescripción de atender exclusivamente a las normas y no a las actitudes y creencias compartidas por quienes participan en el juego del Derecho ha conducido a la teoría kelseniana a algunas situaciones de bloqueo bien conocidas, como la de no poder distinguir entre multas e impuestos.

Confróntense las dos siguientes normas posibles:

N1. A quien supere la velocidad de $120 \mathrm{~km} / \mathrm{h}$ en autopista se le deberá imponer el pago al erario público de 100 euros.

N2. A quien ingrese 30.000 euros anuales se le deberá imponer el pago al erario público de 10.000 euros.

Intuitivamente, todos sabemos que los 100 euros de N1 son una sanción y que no lo son los 10.000 euros de N2. Porque sabemos que el tratamiento desagradable previsto en N1 responde a una conducta ilícita (prohibida) y que eso no es el caso de N2, que también estipula un tratamiento desagradable, pero no como respuesta a una conducta ilícita. Pero para saber esto no es necesario que haya en el Derecho un enunciado del tipo «prohibido ir a más de $120 \mathrm{~km} / \mathrm{h}$ », por una parte, mientras que no hay tal enunciado prohibitivo, o hay un enunciado permisivo, respecto de la conducta consistente en ganar dinero. Bien pudiera ser que no hubiera tales enunciados y que, sin embargo, supiésemos con claridad que la conducta que figura como antecedente del tratamiento desagradable en $\mathrm{N} 1$ es una conducta prohibida (y que la imposición del pago de los 100 euros es, en consecuencia, una multa) y que no pasa otro tanto con N2 (y que la imposición del pago de los 10.000 euros no es, por tanto, una multa sino un impuesto). Y lo sabríamos en virtud de las actitudes compartidas respecto de N1 y N2: a saber, que $\mathrm{N} 1$ trata de disuadir de la conducta correspondiente y que esto no pasa con N2.

\subsubsection{Segunda acotación}

El entender, al modo de KELSEN, que todas las normas jurídicas genuinas o «primarias» están dirigidas a los órganos y ordenan la imposición de sanciones impide ver 
como antinómicas normas que todo el mundo consideraría como tales. Un ejemplo que me gusta mucho es el que encontramos en el libro de MonTESQuieu, Considérations sur les causes de la grandeur des Romains et de leur décadence. Se trata de lo siguiente: el emperador romano Calígula era descendiente a la vez, por vías naturales o de adopción, de Octavio (el que acabaría siendo Augusto) y de Antonio (el lugarteniente de César). Octavio y Antonio se habían enfrentado en la batalla de Accio, en la que triunfó Octavio y fue derrotado Antonio. Pues bien: con ocasión de uno de los aniversarios de la batalla de Accio, Calígula emitió un decreto en el que ordenaba que se castigara a quienes celebraran el aniversario de la batalla de Accio, porque estaban celebrando la derrota de su antepasado Antonio y ordenaba también que se castigara a quienes no celebraran el aniversario de la batalla de Accio porque estaban omitiendo celebrar la victoria de su antepasado Octavio ${ }^{12}$. Estas dos normas aparecen naturalmente como antinómicas si entendemos que ambas están dirigidas a la gente en general y que una de ellas prohíbe, mientras que la otra ordena, celebrar el aniversario de la batalla de Accio. Pero si entendemos que no hay más normas que las dirigidas a los órganos y que las normas no tienen otro contenido que la imposición de sanciones, no hay obviamente antinomia alguna. Los órganos pueden seguir ambas normas sin problema alguno de incompatibilidad: pues la suma de ambas lo que implica es que los órganos deben castigar a todo el mundo. Pero como consecuencia de este enfoque, la función de las normas como guía de la conducta de la gente en general se nos ha evaporado por completo.

\subsubsection{Tercera acotación}

La tercera acotación afecta a la pretensión kelseniana de que su propia contribución estaría situada en una vía media respecto de la «jurisprudencia tradicional», por un lado, y las corrientes del realismo judicialista, por otro. Pero su posición respecto del problema del conflicto entre normas de diferente jerarquía — es decir, la doctrina de la cláusula alternativa tácita - hace que el conjunto de su construcción caiga del lado del realismo judicialista más extremo. Pues, de acuerdo con la doctrina de KELSEN, toda norma - con la única excepción de las normas individuales que ordenan concretos actos de ejecución material, esto es, de las sentencias y resoluciones administrativas - es «norma superior determinante» respecto de otras, es decir, toda norma, con la excepción señalada, regula la producción de otras normas. Pues bien, todas estas normas contienen tácitamente, de acuerdo con KELSEN, una cláusula que autoriza a hacer caso omiso de su contenido expreso. De esta forma, las únicas normas de contenido no tautológico que acaba conteniendo el sistema —esto es, las únicas

${ }^{12}$ El episodio está levemente modificado por mí en relación con el texto de MONTESQUIEU —en el que aparece asimismo una referencia a Drusila que yo he omitido-, para construir a partir de él un ejemplo que resulte pregnante. Lo que leemos en MONTESQUIEU es esto: «Caligula était un vrai sophiste dans sa cruauté. Comme il descendait également d'Antoine et d'Auguste, il disait qu'il punirait les consuls s'ils célébraient le jour de réjonissance établi en memoire de la victoire d'Actium, et qu'il les punirait s'ils ne le celebraient pas. Et, Drusille, à qui il accorda des honneurs divins, étant morte, c'était un crime de la pleurer, parce qu'elle était Déesse, et de ne la pas pleurer, parce qu'elle était sa soeur» (cito por MONTESQUIEU: Considérations sur les causes de la grandeur des Romains et de leur décadence, chronologie et préface par Jean Ehrard, GF-Flammmarion, Paris, 1968, 118-119). 
normas del sistema que pueden cumplir una función de guía de la conducta- son las sentencias y resoluciones administrativas.

\subsubsection{Cuarta acotación}

Esta cuarta acotación tiene que ver con la pretensión kelseniana de que su modelo de ciencia jurídica proporcione una descripción neutral del Derecho. El fracaso de KELSEN a este respecto se hace claro, mostrando que los extraños «enunciados descriptivos de deber ser» que, de acuerdo con KELSEN, serán los propios de la ciencia jurídica, son, en último término, indistinguibles de enunciados normativos plenos que reiteren el deber ser contenido en las normas. Basta recordar, a este respecto, la negativa kelseniana a entender la distinción entre normas y enunciados acerca de ellas en términos de «uso» y «mención», según le proponía HART en un famoso encuentro ${ }^{13}$ : las normas jurídicas, de acuerdo con la propuesta de HART, usarian un lenguaje normativo; lenguaje normativo que aparecería mencionado, pero no usado, en las aserciones de la ciencia jurídica relativas a las normas. Pero esta posibilidad es explícitamente rechazada por KELSEN, quien escribe enfáticamente que «la afirmación [característica de la ciencia jurídica] de que una norma que ordena, autoriza o permite (positivamente) determinada conducta se encuentra "en vigencia" o tiene "validez" [...] sólo puede querer decir que esa conducta debe producirse» ${ }^{14}$. Pero siendo así las cosas, los enunciados de la ciencia jurídica no hacen sino reiterar las prescripciones contenidas en las normas y el programa descriptivista de KELSEN puede considerarse fallido.

\subsection{Lo que está vivo}

KELSEN es el teórico del Derecho más importante del siglo XX, de un lado. De otro, prácticamente todas sus doctrinas importantes deben ser abandonadas, pues llevan a callejones sin salida. ¿Por qué, pues, su importancia? ¿No será toda la teoría de KELSEN un gigantesco error que hay que, sin más, dejar de lado? En mi opinión, de ningún modo son las cosas así. Vale la pena señalar que toda la teoría del Derecho actual es postkelseniana, en un doble sentido: no sólo en el sentido de que no se puede entender sin KELSEN, sino también en el de que sus obras principales han venido construyéndose en diálogo con KELSEN. Esto es particularmente claro en el caso de la gran generación positivista postkelseniana: la ingente obra de BOBBIO, El concepto de Derecho, de HART, o Sobre el Derecho y la justicia, de Ross son textos construidos precisamente en diálogo con KELSEN y frente a KELSEN. Pero también ocurre con las principales manifestaciones del positivismo de hoy, tanto excluyente (en las obras de RAZ, de AlChOURRÓn y Bulygin o de FerRAjoli la presencia de Kelsen es central) como incluyente (piénsese por ejemplo en CARRIÓ). Debe señalarse, sin embargo, que la general limitación del inclusive legal positivism a la provincia cultural angloamericana hace que en él las influencia de KELSEN se perciba casi exclusivamente a través de

${ }_{13}$ H. L. A. HART, «Kelsen Visited» (1963), en HarT, Essays in Jurisprudence and Philosophy, Oxford, Clarendon Press, 1983, 286 y ss.

${ }^{14}$ H. KelsEn, Teoría pura del Derecho, cit., 92. 
HART. Y otro tanto ocurre, como es obvio, en el caso de DwORKIN, principal representante angloamericano del no positivismo contemporáneo. Pero en el caso de otros no positivistas culturalmente más ecuménicos, como ALEXY o NINO, la presencia de KELSEN es, de nuevo, central. Se ha repetido mucho, a propósito de KELSEN, la metáfora de B. DE CHARTRES: si vemos más lejos que él es porque estamos montados sobre sus hombros. Y esto puede afirmarse con entera justicia respecto de cualquiera de las corrientes que podemos distinguir en la actual teoría del Derecho.

La teoría del Derecho de KELSEN es, por otro lado, la primera teoría del Derecho que merece ser calificada precisamente de teoría, esto es, de un cuerpo trabado de proposiciones desde el que se intenta dar respuesta a los problemas que plantea un cierto dominio. Si, como quería SPINOZA, omnis determinatio est negatio, o, como gustaba de repetir POPPER, una teoría es tanto más interesante cuanto más amplio es el campo de lo que niega, la teoría kelseniana aparece inmensamente distanciada, en cuanto a su interés, de lo que eran las doctrinas jurídicas anteriores a ella, que en general estaban constituidas por discursos tan blandos que no negaban casi nada. Y de ahí que las teorías del Derecho posteriores se hayan visto abocadas a ajustar sus cuentas con KELSEN y a construirse a partir de ese ajuste.

Por lo demás, resulta claro que las teorías del Derecho a construir en el presente tienen que distanciarse fuertemente de KELSEN. Las razones de ello son múltiples pero se pueden reducir, en lo esencial, a lo siguiente: una teoría que considera que el concepto de razón práctica es autocontradictorio no puede ser la teoría adecuada para el Derecho del Estado constitucional, pues tal teoría no puede reconocer las pretensiones de fundamentación racional de las decisiones que son características de un sistema jurídico de este tipo. Y para dar cabida a estas pretensiones de fundamentación racional es preciso, en primer lugar, una teoría que no considere que los términos con fuerte carga valorativa que suelen encontrarse en la formulación de, por ejemplo, los principios constitucionales, esto es, los términos que designan conceptos esencialmente controvertidos, son meras puertas abiertas a una discreción judicial irrestricta, sino que caben determinaciones de los mismos racionalmente fundadas. Y que proceder a tales determinaciones implica embarcarse en ejercicios de deliberación moral que han de entenderse como situados dentro de las fronteras de la razón y no más allá de las mismas. Y si una teoría jurídica adecuada para el Derecho del Estado constitucional debe estar abierta a la teoría moral, también debe estarlo a las ciencias sociales. Pues sólo así cabe situar algo más allá del sentido común las polémicas acerca de la eficiencia de diversos cursos de acción alternativos para lograr los diversos objetivos sociales que vienen normativamente ordenados por unas u otras directrices, constitucionales o legislativas.

De forma que la exigencia de autonomía, o de pureza, es una exigencia central de la teoría del Derecho preconizada por KELSEN que debe hoy ser radicalmente abandonada. Pues, a mi juicio, una teoría del Derecho adecuada a las exigencias de los sistemas jurídicos de la hora presente debe ser una teoría del Derecho fuertemente penetrada de teoría ética y de ciencias sociales.

Y tengo también la impresión de que - pero esto lo digo con menos énfasis- la teoría del Derecho a hacer en la actualidad debería renunciar, al menos por el momento, a la pretensión de globalidad de la teoría kelseniana y moverse en ese nivel de 
generalidad más bajo al que J. J. MoRESO, por ejemplo, se ha referido como el de las microteorías ${ }^{15}$. Microteorías que nos permitirían unir argumentativamente en nuestra reflexión análisis conceptuales, examen de normas positivas, cuestiones de teoría de la justicia y cuestiones de diseño institucional. Tal vez orientar nuestra reflexión hacia estas microteorías podría dar a la teoría del Derecho una relevancia para la cultura jurídica, y en general para la cultura pública, mayor de la que hoy tiene.

Pero, si quiere ser culturalmente relevante, la teoría jurídica no puede renunciar a ser precisamente eso, teoría. Y me parece percibir un riesgo de que, frente a las dificultades y el esfuerzo que implica el hacer teoría, y aparentemente justificados por el giro argumentativo que, sin duda, la teoría del Derecho ha experimentado y debe seguir experimentando, se esté imponiendo la tentación de un discurso blando. De un discurso que, bajo el rótulo de análisis de sentencias, se limite a contarnos qué argumentos de las mismas les parecen buenos, malos o regulares a los sedicentes analistas, sin criterios generales a los que esos mismos analistas se sientan vinculados, sin hacer avanzar un ápice nuestra comprensión de nada. En este tipo de discurso, el analista pasa, de una ocasión a otra, de formalista (en uno de los sentidos de esta expresión) a realista, o de formalista (en otro de sus sentidos) a sustantivista, o de partidario de una vinculación literalista del juez a la ley a partidario de un amplio activismo judicial. Y el hilo conductor de todo ello son simplemente las simpatías o antipatías que el analista experimente respecto de las partes de los diferentes procesos. Hoy, como ayer era para KELSEN, el principal adversario de una teoría del Derecho digna de tal nombre es el discurso jurídico que sea puro disfraz de los prejuicios, simpatías y antipatías de sus autores. Y, frente a ello, la lección de rigor kelseniana, sigue siendo, a mi juicio, plenamente actual.

15 J. J. MoRESO, «Cuestiones persistentes y microteorías», en Doxa, núm. 25 (2002): 629-635. 